\title{
Hydrogenation of Olefins Catalyzed by Polymer-Supported Palladium-Imidazole
}

\author{
Udayakumar VELU $^{1}$, Alexander STANISLAUS ${ }^{1}$, Gayathri VIRUPAIAH ${ }^{1, *}$, Shivakumaraiah ${ }^{2}$, \\ Viswanathan BALASUBRAMANIAN ${ }^{3}$ \\ ${ }^{1}$ Department of Studies in Chemistry, Central College Campus, Bangalore University, Bangalore 560 001, India \\ ${ }^{2}$ Siddaganga Institute of Technology, Tumkur, Karnataka 572 103, India \\ ${ }^{3}$ National Centre for Catalysis Research, I.I.T. Madras., Chennai 600 036, India
}

\begin{abstract}
A polymer-supported palladium-imidazole catalyst was used to catalyze the hydrogenation of various olefins under mild conditions. The rate of hydrogenation was studied. The effects of factors such as substrate concentration, catalyst concentration, partial pressure of hydrogen and temperature on initial rate of reaction of selected olefins were investigated. A mechanism for the reaction was proposed from the rate equation. The effects of the solvent and structure of the olefin on the rate of hydrogenation were investigated. The catalyst showed good reusability without any leaching of metal from the support. The homologous analog of the polymer-supported catalyst could not be used as catalyst for the hydrogenation of olefins in methanol because there was precipitation of the metal during reaction.
\end{abstract}

Key words: polymer-supported palladium-imidazole catalyst; cyclohexene; relative reactivity; sequential hydrogenation; recycling efficiency

\section{CLC number: O643 Document code: A}

Many metal complexes that are homogeneous catalysts for various chemical transformations can be bonded onto supports and be used as heterogeneous catalysts [1,2]. The attachment of metal complexes onto a support to make it insoluble prevents the loss of expensive catalyst material and contamination of reaction mixture and product [3]. Such complexes can catalyze various reactions like hydrogenation, oxidation, and Suzuki cross-coupling [4-6]. Environmental concerns continue to encourage the development of these eco-friendly catalysts that work under mild conditions, can be recovered easily, and recycled without much loss of reactivity because they solve many chemical, environmental, and economical problems. Hydrogenation is a useful test reaction widely used in supported metal complex catalysis study [7].

In our previous work, a palladium-imidazole complex bonded to chloromethylated polystyrene beads cross-linked with $6.5 \%$ divinylbenzene was characterized by various techniques and used as catalyst for the hydrogenation of benzylideneaniline and some of its para substituted derivatives under ambient conditions [8]. A polymer-supported palladium-Schiff base complex has been prepared, characterized, and used as catalyst for the hydrogenation of selected olefins [9] and nitroarenes [10].

In the present work, a $\mathrm{Pd}(\operatorname{Imz})_{2} \mathrm{Cl}_{2}(\operatorname{Imz}=$ imidazole $)$ complex was synthesized and used as homogeneous catalyst for the hydrogenation of olefins in methanol under ambient conditions. Precipitation of metal in the reaction mixture was observed during the course of reaction. Hence, it was not a good catalyst for these reactions. The $\mathrm{Pd}(\operatorname{Imz})_{2} \mathrm{Cl}_{2}$ complex was insoluble and stable when bonded to a polymer support. The polymer supported palladium-imidazole complex was used as a heterogeneous catalyst for the hydrogenation of various olefins under mild conditions. Kinetic studies were performed on selected olefins. On the basis of the kinetic study and rate equation, a reaction mechanism was proposed. The catalyst showed good reusability without any leaching of metal from the support.

\section{Experimental}

\subsection{Synthesis of the catalyst}

Chloromethylated polystyrene beads cross-linked with $6.5 \%$ divinylbenzene was donated by Thermax India Ltd., Pune, India. The solvents and all other reagents were purified before use.

The polymer-supported palladium-imidazole complex (Scheme 1) was prepared by the procedure described in our earlier publication [8]. The $\mathrm{Pd}(\mathrm{Imz})_{2} \mathrm{Cl}_{2}$ complex was pre-

Received 19 September 2010. Accepted 11 November 2010.

*Corresponding author. Tel: +91-080-2296 1348; E-mail: gayathritvr@yahoo.co.in

Foundation item: Supported by University Grants Commission, Government of India.

English edition available online at ScienceDirect (http://www.sciencedirect.com/science/journal/18722067). 


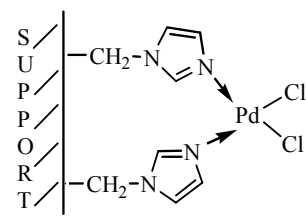

Scheme 1. Polymer-supported palladium-imidazole complex.

pared according to a literature report [11].

\subsection{Hydrogenation of olefins}

In a typical hydrogenation experiment, the catalyst was added along with the solvent into a glass reactor, flushed with hydrogen and evacuated. Water was circulated at a desired temperature from a thermostat through the outer jacket of the reactor and that of the hydrogen gas burette. A known quantity of the substrate was injected and the system was opened to the hydrogen gas burette. The reactions were monitored by the hydrogen uptake at set intervals of time. At the end of the each reaction, the catalyst was separated by filtration and the products were identified by GC and GC-MS techniques. A blank reaction was also done in the absence of catalyst. GC data were obtained with a Shimadzu GC with BP5 capillary column. GC-MS data were obtained using a Shimadzu GC-MS QP5050A with BP5 column.

\section{Results and discussion}

The initial rates of hydrogenation of the various olefins were determined from the slope of the plot of volume of hydrogen uptake as a function of time. The effects of various parameters on the rate of reaction were investigated.

\subsection{Effect of solvent on the rate of hydrogenation of cyclohexene}

Polarity, coordinating ability of the solvent with the catalyst, capacity to swell the polymer support, and hydrogen solubility in the solvent can influence the rate of hydrogenation. To study the effect of solvents, hydrogenation of cyclohexene was carried with constant concentrations of $[$ Cyclohexene $]=6.67 \times 10^{-2} \mathrm{~mol} / \mathrm{L}$ and $[$ Catalyst $]=5.92 \times$ $10^{-3} \mathrm{~mol} / \mathrm{L}$ (calculated based on $\mathrm{Pd}$ ), at temperature $=30{ }^{\circ} \mathrm{C}$, $79.5 \mathrm{kPa}$ hydrogen pressure using $30 \mathrm{ml}$ of different solvents (Table 1). The results revealed that the polar solvents favored the hydrogenation reaction. Methanol, which is polar and has good hydrogen solubility [12], shows the highest rate of reaction. Better swelling of the supported catalyst in methanol can be another reason for the high rate of reaction $[8,13]$. Dimethylformamide and dimethyl sulfoxide, although polar, can coordinate strongly and irre-
Table 1 Hydrogenation of cyclohexene in various solvents

\begin{tabular}{lc}
\hline Solvent & Initial rate $\times 10^{-4}(\mathrm{~mol} /(\mathrm{L} \cdot \mathrm{min}))$ \\
\hline Methanol & 9.56 \\
Ethanol & 9.38 \\
Toluene & 5.68 \\
Tertahydrofuran & 5.26 \\
Acetone & 5.07 \\
Benzene & 3.38 \\
Dimethyl sulfoxide & 0.96 \\
Dimethylformamide & 0.89 \\
$75 \%$ methanol-25\% benzene & 8.96 \\
$50 \%$ methanol-50\% benzene & 7.91 \\
$25 \%$ methanol-75\% benzene & 6.86 \\
\hline
\end{tabular}

versibly with the catalyst and hindered the coordination of hydrogen and olefin with the catalyst, resulting in very low rates of reaction. Since a higher reaction rate was observed in methanol, further studies were carried out with methanol. The effect of increased benzene concentration in the reaction medium was also studied. The results are included in Table 1 [14-16].

\subsection{Reaction kinetics and mechanism}

Kinetic studies were carried out using cyclohexene (cyclic olefin), diethylmaleate (DEM), and diethylfumarate (DEF) (internal olefin, geometric isomers) as substrates.

\subsubsection{Effect of catalyst concentration on the rate of hydrogenation}

The order of reaction, calculated from the slope of the plot of $\log$ (Initial rate) against $\log$ [Catalyst], revealed that the initial rate of hydrogenation for all the substrates was first order in catalyst concentration in the range $1.46 \times 10^{-3}$ to $7.40 \times 10^{-3} \mathrm{~mol} / \mathrm{L}$, at constant [Substrate] $=6.67 \times 10^{-2}$ $\mathrm{mol} / \mathrm{L}, 79.5 \mathrm{kPa}$ hydrogen pressure, temperature $=30{ }^{\circ} \mathrm{C}$, and with $30 \mathrm{ml}$ methanol (Fig. 1) [16].

\subsubsection{Effect of substrate concentration on the rate of hydrogenation}

The effect of substrate concentration on the rate of reaction was studied by varying the substrate concentration in the range $3.3 \times 10^{-2}$ to $16.7 \times 10^{-2} \mathrm{~mol} / \mathrm{L}$ at constant [Catalyst $]=5.92 \times 10^{-3} \mathrm{~mol} / \mathrm{L}$, temperature $=30^{\circ} \mathrm{C}, 79.5 \mathrm{kPa}$ pressure of hydrogen, and with $30 \mathrm{ml}$ methanol. The slope obtained from the plot of $\log$ (Initial rate) against $\log$ [Substrate] revealed that the reaction followed first order kinetics in the substrate concentration range $3.3 \times 10^{-2}$ to $1.0 \times 10^{-1} \mathrm{~mol} / \mathrm{L}$ (Fig. 2). Above this range, the initial rate of reaction followed fractional order $[16,17]$. This can be 


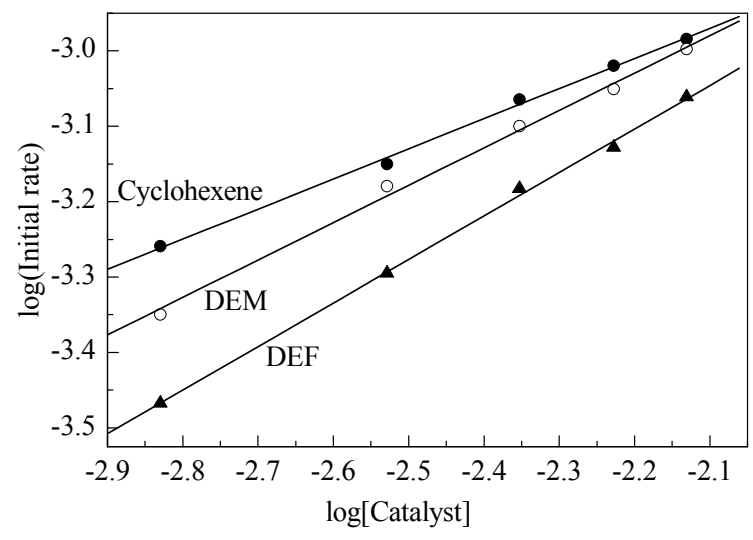

Fig. 1. Dependency of initial rate of hydrogenation on catalyst concentration.

attributed to the fact that as the catalyst concentration was constant, all the catalyst would have been used by the substrate and there was insufficient catalyst at higher concentrations of the substrate.

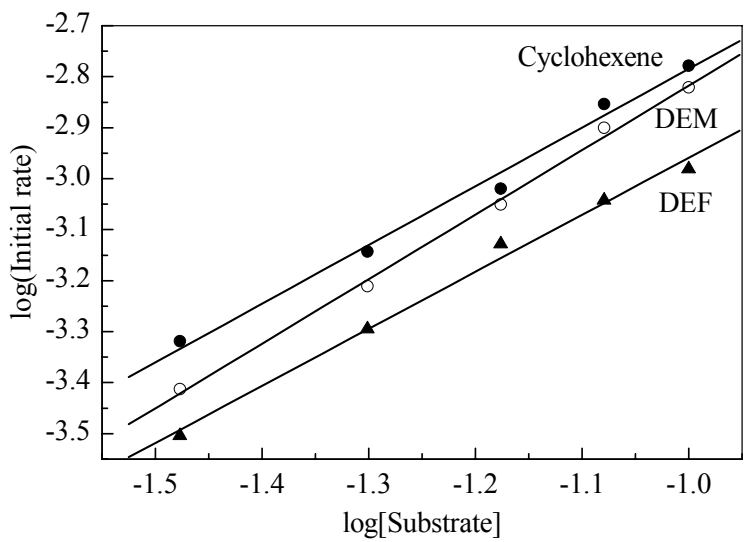

Fig. 2. Dependence of initial rate of hydrogenation on substrate concentration.

\subsubsection{Effect of partial pressure of hydrogen on the rate of hydrogenation}

A range of partial pressures of hydrogen was obtained by mixing pure nitrogen and hydrogen in a gas burette with the total pressure of the mixture of gases as $1.0 \times 10^{5} \mathrm{~Pa}$. The concentration of dissolved hydrogen in the solvent was calculated using Henry's law [14,18]. Hydrogenation was carried out in the hydrogen concentration range $1.52 \times 10^{-3}$ to

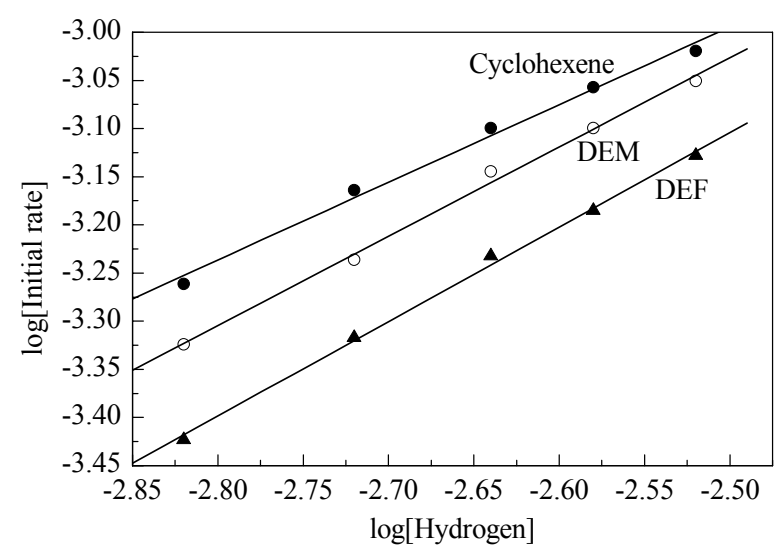

Fig. 3. Dependency of initial rate of hydrogenation on partial pressure of hydrogen.

$3.04 \times 10^{-3} \mathrm{~mol} / \mathrm{L}$ with constant [Catalyst] $=5.92 \times 10^{-3}$ $\mathrm{mol} / \mathrm{L},[$ Substrate $]=6.67 \times 10^{-2} \mathrm{~mol} / \mathrm{L}$, temperature $=30$ ${ }^{\circ} \mathrm{C}, 30 \mathrm{ml}$ methanol. The order of reaction obtained from the slope of the plot of $\log$ (Initial rate) against $\log$ [Hydrogen] was first order (Fig. 3) [19].

On the basis of the kinetic studies, a reaction mechanism (Scheme 2) and rate equation (Eq. (1)) were proposed [18]. In this proposed mechanism, the alkene initially forms a $\pi$-complex intermediate in a reversible manner with the polymer-supported catalyst. This intermediate reacts with hydrogen to form a dihydrido complex to subsequently form an alkyl-palladium complex that then undergoes reductive elimination reaction to yield the alkane.

$$
\text { Rate }=\frac{K k_{1}[\text { Catalyst }][\text { Substrate }]\left[\mathrm{H}_{2}\right]}{1+K[\text { Substrate }]}
$$

The deduced rate equation (Eq. (1)) described the experimental data reasonably well. In Eq. (1), $K$ is the equilibrium constant for the reversible reaction, and $k_{1}$ is the rate constant for the rate determining step. Equation (1) is consistent with that the reaction follows first order with catalyst concentration and partial pressure of hydrogen, and follows first order with respect to substrate concentration in its lower concentration range and fractional order at higher substrate concentrations. The lines in Fig. 1 and Fig. 2 are parallel, which revealed that cyclohexene (cyclic olefin), DEM, and DEF (linear internal olefins, geometric isomers) follow the same reaction mechanism for hydrogenation (Scheme 2) under the studied conditions.

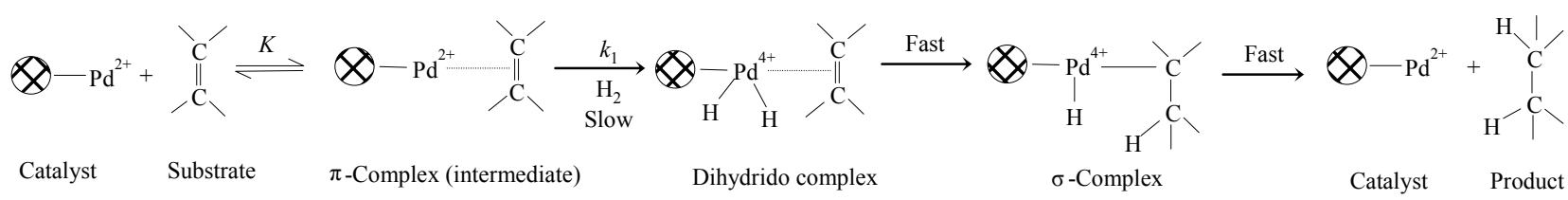

Scheme 2. Mechanism for the hydrogenation. 


\subsubsection{Effect of temperature on the rate of hydrogenation}

Hydrogenation reactions were carried out in the temperature range 30 to $50{ }^{\circ} \mathrm{C}$ with constant [Catalyst] $=5.92 \times 10^{-3}$ $\mathrm{mol} / \mathrm{L}$, [Substrate] $=6.67 \times 10^{-2} \mathrm{~mol} / \mathrm{L}, 79.5 \mathrm{kPa}$ pressure of hydrogen, and $30 \mathrm{ml}$ methanol. An increase in the rate of reaction with temperature was observed. The activation energies found from the Arrhenius plot (Fig. 4) and activation entropy are tabulated in Table 2 [16]. The negative activation entropy values indicated that the adsorption of the substrate on the catalyst surface resulted in the loss of three translational degrees of freedom and the adsorbed complex probably had only three vibrational degrees of freedom. This type of interaction may facilitate hydrogenation.

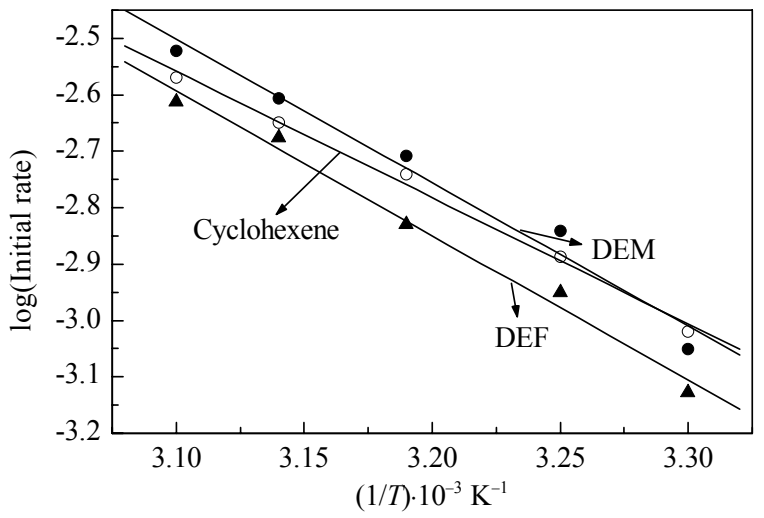

Fig. 4. Arrhenius plot for the hydrogenation reaction.

Table 2 Activation energy and activation entropy for the hydrogenation reaction

\begin{tabular}{lcc}
\hline Substrate & $\begin{array}{c}\text { Activation } \\
\text { energy }(\mathrm{kJ} / \mathrm{mol})\end{array}$ & $\begin{array}{c}\text { Activation entropy } \\
(\mathrm{J} /(\mathrm{K} \cdot \mathrm{mol}))\end{array}$ \\
\hline Cyclohexene & 42 & -170 \\
DEM & 48 & -150 \\
DEF & 49 & -152 \\
\hline
\end{tabular}

\subsection{Effect of olefin structure on its reactivity for hydrogenation}

The structure, position of the double bond, and substituents around the double bond of the olefin have a remarkable influence on the hydrogenation reactivity. To study the influence of the structure of olefin on the rate of hydrogenation, some open-chain and cyclic olefins were used as substrate. 1-Hexene, 1-heptene, 1-octene (open-chain terminal olefins), diethylmaleate, diethylfumarate (open-chain internal olefins with carbon-carbon double bond in conjugation with two ester groups, geometric isomers), ethyloleate (internal olefin with long chain), cyclohexene, cyclooctene (cyclic olefins), 1,5-cyclooctadiene, 2,5-norbornadiene (non-conjugated cyclic dienes), 1,3-cyclooctadiene (conju- gated cyclic diene) were chosen as substrates. The relative activities of the various olefins and their initial rates of hydrogenation were studied under identical conditions: [Substrate $]=6.67 \times 10^{-2} \mathrm{~mol} / \mathrm{L},[$ Catalyst $]=5.92 \times 10^{-3} \mathrm{~mol} / \mathrm{L}$, $79.5 \mathrm{kPa}$ hydrogen pressure, at $30{ }^{\circ} \mathrm{C}$ temperature and with $30 \mathrm{ml}$ methanol. The data are listed in Table 3.

Table 3 Relative reactivities of the olefins for the hydrogenation reaction

\begin{tabular}{lcc}
\hline Substrate & $\begin{array}{c}\text { Initial rate } \times 10^{-4} \\
(\mathrm{~mol} /(\mathrm{L} \cdot \mathrm{min}))\end{array}$ & $\begin{array}{c}\text { Relative } \\
\text { reactivity }^{*}\end{array}$ \\
\hline 1-Hexene & 11.6 & 100 \\
1-Heptene & 9.7 & 84 \\
1-Octene & 7.5 & 65 \\
Cyclohexene & 9.5 & 82 \\
Cyclooctene & 5.6 & 49 \\
Ethyloleate & 9.1 & 79 \\
Diethylmaleate & 8.9 & 77 \\
Diethylfumarate & 7.4 & 64 \\
2,5-Norbornadiene & 12.5 & 108 \\
1,5-Cyclooctadiene & 8.7 & 75 \\
1,3-Cyclooctadiene & 10.1 & 87 \\
\hline
\end{tabular}

*Using initia1 rate of hydrogenation of 1-hexene $=100$.

The reactivity of the olefins (Table 3 ) can be interpreted on the basis of the complex formation ability of the olefin with the catalyst (Scheme 2) [20]. The structure, position of the double bond, and substituents around the double bond of the olefin can influence its ability to form a complex with the catalyst, which determines the reactivity.

\subsubsection{Effect of olefin chain length}

The increase in the chain length of the olefin did not facilitate activating the olefin double bond, but caused steric hindrance in forming the complex with the catalyst and thereby caused a decrease in reactivity. The hydrogenation reactivity followed the order: 1-hexene $>1$-heptene $>$ 1-octene [17]. This may be due to that the electronic inductive effect is not significant beyond three $\sigma$-bonds in the olefin chain.

\subsubsection{Effect of olefin ring size}

Hydrogenation of cyclohexene and cyclooctene were carried out to establish the influence of ring size on reactivity. The increase in ring size of a cyclic olefin decreased the rate of hydrogenation, as reflected in the relative reactivity. The lower activity of cyclooctene was attributed to the larger ring size, as a larger ring size decreases the complexing ability of the cyclooctene with the catalyst due to steric hindrance. Different authors using various catalysts have reported different reactivity $[21,22]$. The order of reactivity 
1-hexene $>$ cyclohexene $>1$-octene $>$ cyclooctene revealed that the reactivity of olefins decreases on moving from linear terminal alkenes to cyclic olefins.

\subsubsection{Effect of position of the carbon-carbon double bond in the alkene chain}

The position of the carbon-carbon double bond in the alkene chain can affect its reactivity. An internal double bond in the olefin has a larger steric hindrance for complex formation with the catalyst. Hence the hydrogenation of an internal olefin like ethyloleate is more difficult than that of a terminal olefin like 1-hexene.

\subsubsection{Reaction with cis-trans isomers}

On the basis of symmetry, one would expect cis alkenes to possess more basic pi character than their trans isomer. Cis-isomer diethylmaleate was hydrogenated faster than trans-isomer diethylfumarate [23].

\subsubsection{Reactivity of dienes}

Dienes may form chelate complexes with the catalyst and the reactivity of these dienes depends on the ability of the diene to form a complex with the catalyst. In case of the non-conjugated dienes 1,5-cyclooctadiene and 2,5-norbornadiene (NBD), the relative reactivity was a function of ring strain. The higher double bond strain in NBD facilitates easy complex formation with the catalyst and promotes hydrogenation. GC and GC-MS analysis of hydrogenation of NBD revealed the sequential hydrogenation of its double bonds (Fig. 5) [24,25] and the reaction was very fast at the beginning. The initial stage of reaction was more selective to norbornene (NBE) and very small

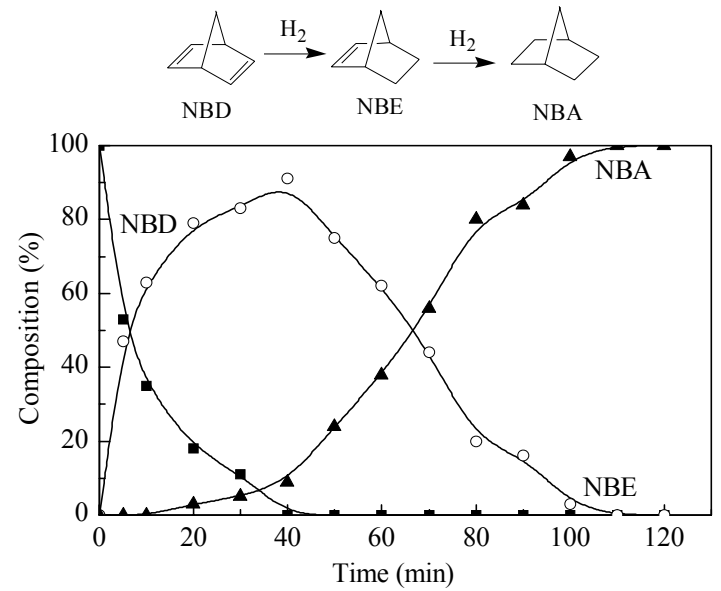

Fig. 5. Sequential hydrogenation of NBD. [Catalyst] $=5.92 \times 10^{-3}$ $\mathrm{mol} / \mathrm{L},[\mathrm{NBD}]=6.67 \times 10^{-2} \mathrm{~mol} / \mathrm{L}, 79.5 \mathrm{kPa}$ hydrogen pressure, temperature $=30^{\circ} \mathrm{C}, 30 \mathrm{ml}$ methanol. amount of norbornane (NBA) was observed in the reaction mixture. Subsequently, NBE was hydrogenated to NBA only when most of the NBD was converted to NBE. This high selectivity for NBD may be due to selective adsorption and/or easy complex formation of NBD with the catalyst. The conjugated diene 1,3-cyclooctadiene was hydrogenated at a faster rate relative to non-conjugated 1,5-cyclooctadiene.

The conversions of various olefins obtained by GC under the reaction conditions of $\left[\right.$ Substrate] $=6.67 \times 10^{-2} \mathrm{~mol} / \mathrm{L}$, $[$ Catalyst $]=5.92 \times 10^{-3} \mathrm{~mol} / \mathrm{L}, 30 \mathrm{ml}$ methanol, $30{ }^{\circ} \mathrm{C}$ temperature, $79.5 \mathrm{kPa}$ hydrogen pressure are tabulated in Table 4.

Table 4 Percentage conversion of various olefins for the hydrogenation reaction

\begin{tabular}{lcc}
\hline Substrate & Product & Conversion $^{*}(\%)$ \\
\hline 1-Hexene & $n$-hexane & 97 \\
1-Heptene & heptane & 82 \\
1-Octene & octane & 96 \\
Cyclohexene & cyclohexane & 91 \\
Cyclooctene & cyclooctane & 97 \\
Ethyloleate & ethyl octadecanoate & 90 \\
Diethylmaleate & diethyl succinate & 82 \\
Diethylfumarate & diethyl succinate & 95 \\
2,5-Norbornadiene & norbornane & 96 \\
1,5-Cyclooctadiene & cyclooctane & 97 \\
1,3-Cyclooctadiene & cyclooctane & 96 \\
\hline
\end{tabular}

${ }^{*}$ As per GC analysis.

\subsection{Recycling efficiency of the catalyst}

The catalyst was recycled six times using cyclohexene as substrate with constant [Cyclohexene $]=6.67 \times 10^{-2} \mathrm{~mol} / \mathrm{L}$, $[$ Catalyst $]=5.92 \times 10^{-3} \mathrm{~mol} / \mathrm{L}, 30 \mathrm{ml}$ methanol, $30{ }^{\circ} \mathrm{C}$ temperature, and $79.5 \mathrm{kPa}$ hydrogen pressure. The initial rate remained almost constant over six cycles without significant loss of catalytic activity (Table 5). The palladium content of the catalyst estimated using an atomic absorption spectrophotometer after six cycles was found to be almost the same as that of the fresh catalyst. When an estimate of metal content was carried out for the reaction mixture at the end of each cycle, no metal was present in the solution, revealing

Table 5 Recycling efficiency of the catalyst

\begin{tabular}{cc}
\hline Cycle & Initial rate $\times 10^{-4}(\mathrm{~mol} /(\mathrm{L} \cdot \mathrm{min}))$ \\
\hline Fresh & 9.56 \\
1 & 9.55 \\
2 & 9.55 \\
3 & 9.54 \\
4 & 9.54 \\
5 & 9.53 \\
6 & 9.52 \\
\hline
\end{tabular}


no metal leaching from the support.

\section{Conclusions}

A polymer-supported palladium-imidazole complex is a promising catalyst for the hydrogenation of various olefins under mild conditions. The solvent used and structure of the olefin played a dominant role on the reactivity. The ability of the olefin to form a complex with the catalyst during hydrogenation provided the basis for interpreting the relative reactivity of the olefins. Sequential hydrogenation of the double bonds was observed in the case of 2,5-norbornadiene. The catalyst was easily recovered and reusable, thus it is environment friendly.

\section{Acknowledgements}

Authors wish to thank University Grants Commission for Departmental research support and Department of science and technology for Fund for improvement of science technology programme, Thermax India Ltd., Pune, India, Sophisticated testing instrumentation centre, Cochin and Central dug research institute, Lucknow. Udayakumar VELU thanks Sree Siddaganga Education Society, Sree Siddaganga Math, Tumkur, Karnataka, India, for extending support for doctoral studies.

\section{References}

1 Candierno V, Crochet P, Diez J, Garcia-Alvarez J, GarciaGarrido S E, Garcia-Granda S, Gimeno J, Rodriguez M A. Dalton Trans, 2003: 3240

2 Corma A, Garcia H. Top Catal, 2008, 48: 8

3 Bonds W D Jr, Brubaker C H Jr, Chandrashekaran E S, Gibbons C, Grubbs R H, Kroll L C. J Am Chem Soc, 1975, 97: 2128
4 Trakarnpruk W, Kanjina W. Thammasat Int J Sci Technol, 2008, 13: 1

5 Islam M, Mondal P, Mondal S, Mukherjee S, Roy A S, Mubarak M, Paul M. J Inorg Organomet Polym, 2010, 20: 87

6 Islam S M, Mondal P, Tuhina K, Roy A S, Mondal S, Hossain D. J Inorg Organomet Polym, 2010, 20: 264

7 Islam M, Mondal P, Roy A S, Tuhina K. Transition Met Chem, 2010, 35: 427

8 Udayakumar V, Alexander S, Gayathri V, Shivakumaraiah, Patil K R, Viswanathan B. J Mol Catal A, 2010, 317: 111

9 Alexander S, Udayakumar V, Gayathri V. J Mol Catal A, 2009, 314: 21

10 Alexander S, Udayakumar V, Nagaraj N, Gayathri V. Transition Met Chem, 2010, 35: 247

11 Van Karalingen C G, De Ridder J K, Reeduk J. Inorg Chim Acta, 1979, 36: 69

12 Welton T. Chem Rev, 1999, 99: 2071

13 Terasawa M, Kaneda K, Imanaka T, Teranishi S. J Catal, 1978, 51: 406

14 Nayak S D, Mahadevan V, Srinivasan M. J Catal, 1985, 92 327

15 Bruner H, Bailer J C Jr. Inorg Chem, 1973, 12: 1465

16 John J, Dalal M K, Patel D R, Ram R N. J Macromol Sci Part A Pure Appl Chem A, 1997, 34: 489

17 Ramesh B, Sadanand D T, Reddy S G, Swamy K V, Saiprakash P K. Transition Met Chem, 2000, 25: 639

18 Jainamma M, Mahadevan V. J Mol Catal, 1990, 60: 189

19 Parameswaran V R, Vancheesan S. React Kinet Catal Lett, 1991, 45: 55

20 Yuffa A Y, Lisichkin G V. Russ Chem Rev, 1978, 47: 751

21 Traynham J G, Sehnert M F. J Am Chem Soc, 1956, 78: 4024

22 Campelo J M, Garacia A, Luna D, Marinas J M. Appl Catal, 1984, 10: 1

23 Sikorski J A, Brown H C. J Org Chem, 1982, 47: 872

24 Amir-Ebrahimi V, Rooney J. Catal Lett, 2009, 127: 20

25 Fahey D R. J Org Chem, 1973, 38: 3343 